Pacific Journal of Mathematics

A REMARK ON TONELLI'S THEOREM ON INTEGRATION IN 


\section{A REMARK ON TONELLI'S THEOREM ON INTEGRATION IN PRODUCT SPACES}

\section{A. Mukherjea}

This paper is concerned to show a connection between the validity of Tonelli's theorem on integration in the product of two measure spaces and the semifiniteness of the product measure. The classical Tonelli theorem is usually stated in a sigma-finite setting. It is shown in this paper, among other things, that in a product measure space, where one of the measures is sigma-finite and other one semifinite (not necessarily sigma-finite), Tonelli's theorem is valid only if the product measure is semifinite and on the other hand, if the product of any two measures is semifinite, then Tonelli's theorem is valid.

1. Let $\left(X, U, \beta_{1}\right)$ and $\left(Y, V, \beta_{2}\right)$ be any two arbitrary measure spaces where $U$ and $V$ are sigma-algebras of subsets of $X$ and $Y$, respectively, and $\beta_{1}$ and $\beta_{2}$ are two nonnegative measures on $U$ and $V$ respectively. Let $U \times V$ be the smallest sigma-algebra containing all the measurable rectangles of $X \times Y$. The product measure $\beta_{1} \times \beta_{2}$ (we call it $\beta^{*}$, for simplicity) is the restriction to $U \times V$ of the outer measure induced by the measure $\beta$ on the algebra $W$ consisting of the measurable rectangles of $X \times Y$ and their finite disjoint unions where for every measurable rectangle $P \times Q, \beta(P \times Q)=\beta_{1}(P) \beta_{2}(Q)$. (See [4], p. 254). $\quad \beta_{1}$ is called semifinite if given $A$ in $U$ with $\beta_{1}(A)=$ $\infty$, we can find $B$ in $U, B \subset A$ and $0<\beta(B)<\infty$. This definition, which at first glance seems to be less restricted than semifiniteness as defined in [4], p. 220, is actually equivalent to Royden's definition, as Lemma 1 in the next section shows. Every sigma-finite measure is semifinite, but not conversely. (For example, consider any non-sigmafinite regular Borel measure on a locally compact space or a counting measure on an uncountable set). The product measure $\beta_{1} \times \beta_{2}$ may not be semifinite even when $\beta_{1}$ is sigma-finite and $\beta_{2}$ semifinite, as Example 1 shows. For the purpose of reference, let us state the following two well-known Theorems in a form, which is slightly different from that given in [2] or [4].

Fubini's Theorem. Let $f(x, y)$ be $\beta^{*}$-integrable on $U \times V$. Then both the iterated integrals of $f$ are well-defined and

$$
\int f d \beta^{*}=\iint f d \beta_{1} d \beta_{2}=\iint f d \beta_{2} d \beta_{1} \text {. }
$$


The proof of this theorem follows easily from Theorem C, p. 147 in [2], if we observe the following points. First, since $f$ is $\beta^{*}$-integrable on $U \times V$, the set

$$
E=\{(x, y) \in X \times Y: f(x, y) \text { is nonzero }\}
$$

is sigma-finite with respect to $\beta^{*}$. Second, it follows from Proposition 6 on p. 256 in [4] that we can find a measurable rectangle $P \times Q$ such that $E \subset P \times Q$ where $P$ and $Q$ are sigma-finite with respect to $\beta_{1}$ and $\beta_{2}$ respectively.

TONELli's TheOREm. Let $\beta_{1}$ and $\beta_{2}$ be both sigma-finite. Let $f(x, y)$ be a nonnegative $U \times V$ measurable function. If one of the iterated integrals of $f$ is well-defined and finite, then the other one is also and

$$
\int f d \beta^{*}=\iint f d \beta_{1} d \beta_{2}=\iint f d \beta_{2} d \beta_{1}
$$

This Theorem is precisely Theorem B in [2], p. 147.

The main purpose of our note is to present the following version of Tonelli's Theorem, which certainly is more informative than the classical version and tells us more about product integration of nonnegative measurable functions. The proof of this Theorem is given in the next section.

THEOREM 1. Suppose one of $\beta_{1}$ and $\beta_{2}$ is sigma-finite and the other one is semifinite. Then Tonelli's Theorem is valid if and only if $\beta^{*}$ is semifinite. The sigma-finiteness assumption can be replaced by semifiniteness if we assume one of the following two conditions:

(a) for all $U \times V$ measurable sets $A$, the function $\beta_{1}\left(A^{y}\right)$ is measurable, where $A^{y}=\{x:(x, y) \in A\}$;

(b) for all $U \times V$ measurable sets $A$, the function $\beta_{1}\left(A_{x}\right)$ is measurable, where $A_{x}=\{y:(x, y) \in A\}$.

Berberian in [1] defines product measure in a different way. In $\S 3$, we show that for his product measure, Fubini's theorem does not hold for non-sigma-finite $\beta_{1}$ and $\beta_{2}$ and only a part of Tonelli's theorem holds. This answers, at least partly, a question of Berberian [2, Problem 4, p. 144].

Finally, we take this opportunity to record our thanks to the referee for his useful comments and also for pointing out that Example 1, Lemma 2, with a slightly different proof, and Proposition 1 (only its first sentence) appeared in [3].

2. First, we consider an Example showing that $\beta^{*}$ need not be 
semifinite even when $\beta_{1}$ is sigma-finite and $\beta_{2}$ is semifinite.

EXAMPLE 1. Let $X=Y=[0, \infty)$ and $U=V=$ the Lebesgue measurable subsets of $[0, \infty)$. Let $\beta_{1}$ be the Lebesgue measure and $\beta_{2}$ be the counting measure. Let $D$ be the diagonal of $X \times Y$. Then $\iint I_{D}(x, y) d \beta_{1} d \beta_{2}=0$ and $\iint I_{D}(x, y) d \beta_{2} d \beta_{1}=\infty$, where $I_{D}$ is the characteristic function of $D$. Hence $\beta^{*}(D)$ is $\infty$, since otherwise, by Fubini's Theorem, the iterated integrals will be equal. If $D_{1} \subset D, D_{1} \in U \times V$ and $\beta^{*}\left(D_{1}\right)<\infty$, then by Fubini's Theorem,

$$
\beta^{*}\left(D_{\tau}\right)=\iint I_{D_{1}}(x, y) d \beta_{1} d \beta_{2}=0 .
$$

Hence $\beta^{*}$ is not semifinite.

The proof of Theorem 1 will follow from the following four Lemmas.

Lemma 1. Let $\beta_{1}$ be semifinite. Then given $B \in U$ with $\beta_{1}(B)=$ $\infty$ and any positive integer $n$, there exists $C \in U, C \subset B$ and $n<$ $\beta_{1}(C)<\infty$.

Proof. Let $T$ be the family of all collections $Q$ of $\beta_{1}$ measurable subsets of $B$ with finite positive $\beta_{1}$-measure such that any two distinct sets in $Q$ are disjoint. Then clearly $T$ is nonempty, since $\beta_{1}$ is semifinite. We partially order the collections $Q$ in $T$ by inclusion. Every linearly odered subset of $T$ has a upper bound, namely the union of all the collections in this subset. Therefore, by Zorn's Lemma, this set $T$ has a maximal element, say $Q_{0}$. If $Q_{0}$ is a countable collection, then we must have $\beta_{1}\left(\cup_{A \in Q_{0}} A\right)=\infty$; for, otherwise, we can find $D \subset B-\bigcup_{A \in Q_{0}} A$ with $0<\beta_{1}(D)<\infty$ and then $Q_{0} \cup\{D\}$ will contradict the maximality of $Q_{0}$. The Lemma then follows clearly when $Q_{0}$ is countable. Now let $Q_{0}$ be uncountable. Let us define $Q_{0 n}=\left\{A \in Q_{0}\right.$ : $\left.\beta_{1}(A)>1 / n\right\}$. Then for some positive integer $m, Q_{0 m}$ is uncountable. From this observation, the Lemma is clear again.

Lemma 2. Let $\beta^{*}$ be semifinite. Then Tonelli's Theorem is valid.

Proof. Suppose $f(x, y)$ is a nonnegative $U \times V$-measurable function such that $\iint f(x, y) d \beta_{1} d \beta_{2}$ is well-defined and equal to some nonnegative-number $k<\infty$. We claim that the support of $f$ is $\beta^{*}$-sigma-finite. If our claim is false, we can find a positive integer $n$ such that if

$$
A_{n}=\{(x, y\}: f(x, y)>1 / n\},
$$

then $\beta^{*}\left(A_{n}\right)=\infty$. Since $\beta^{*}$ is semifinite, we can find $B \subset A_{n}, B \in$ 
$U \times V$ and $2 k n<\beta^{*}(B)<\infty$, by Lemma 1 . Then by Fubini's Theorem,

$$
2 k<\beta^{*}(B) / n=\iint 1 / n . \quad I_{B}(x, y) d \beta_{1} d \beta_{2} \leqq \iint f d \beta_{1} d \beta_{2}
$$

which is a contradiction. Hence, the support of $f$ is sigma-finite so that we can find a measurable rectangle $P \times Q$ containing the support of $f$ such that $P$ and $Q$ are sigma-finite with respect to $\beta_{1}$ and $\beta_{2}$ respectively. (This can be done using Proposition 6 on p. 256 in [4]). Now the Lemma follows from the classical Tonelli Theorem.

Lemma 3. Let $\beta_{1}$ be sigma-finite and $\beta_{2}$ semifinite. If Tonelli's Theorem is valid, then $\beta^{*}$ is semifinite.

Proof. Let $A$ be a $U \times V$ measurable set such that $\beta^{*}(A)$ is infinite. Since $\beta_{1}$ is sigma-finite, $\beta_{1}\left(A^{y}\right)$ is a measurable function of $y$. (This follows from the proof of the classical Tonelli theorem). Therefore, by hypothesis,

$$
\iint I_{A}(x, y) d \beta_{1} d \beta_{2}=\int_{S} \beta_{1}\left(A^{y}\right) d \beta_{2}=\infty,
$$

where $S=\left\{y: \beta_{1}\left(A^{y}\right)>0\right\}$.

We separate the proof into two distinct cases.

Case 1. Suppose $\beta_{2}(S)<\infty$.

Subcase (i). Suppose $\beta_{2}(B)=2 p>0$, where

$$
B=\left\{y: \beta_{1}\left(A^{y}\right)=\infty\right\} .
$$

Since $\beta_{1}$ is sigma-finite, there are sets $C_{i}$ in $U$ such that $C_{i} \subset C_{i+1}$, $X=\mathbf{U}_{i=1} C_{i}$ and $\beta_{1}\left(C_{i}\right)<\infty$ for every positive integer $i$. Now $\beta_{1}\left(C_{i} \cap A^{y}\right)$ is a measurable function of $y$ since by the sigma-finiteness of $\beta_{1}$, the function $\int I_{A}(x, y) I_{C_{i}}(x) d \beta_{1}$ is measurable. Since for any arbitrary positive number $k$,

$$
B \subset \bigcup_{i=1}^{\infty}\left\{y: \beta_{1}\left(C_{i} \cap A^{y}\right)>k\right\},
$$

there exist an $i$ and $D$ in $V$ such that

$$
p<\beta_{2}(D)<\infty \text { and } D \subset\left\{y: \beta_{1}\left(C_{i} \cap A^{y}\right)>k\right\} \text {. }
$$

Let $E=A \cap\left(C_{i} \times D\right)$. Then by Fubini's theorem,

$$
0<k p<\int_{D} \beta_{1}\left(C_{i} \cap A^{y}\right) d \beta_{2}=\beta^{*}(E) \leqq \beta_{1}\left(C_{i}\right) \beta_{2}(D)<\infty \text {. }
$$


Subcase (ii). Suppose $\beta_{2}(B)=0$, where $B$ is as above. Now if $C_{n}=\left\{y: n<\beta_{1}\left(A^{y}\right) \leqq n+1\right\}$, then $S=\bigcup_{n=0}^{\infty} C_{n}$. Therefore,

$$
\sum_{n=0}^{\infty} n \beta_{2}\left(C_{n}\right) \leqq \int_{S} \beta_{1}\left(A^{y}\right) d \beta_{2}=\infty=\sum_{n=0}^{\infty}(n+1) \beta_{2}\left(C_{n}\right) \text {. }
$$

Since $\beta_{2}(S)<\infty$ and $\beta_{2}$ is semifinite, given an arbitrary positive number $m$, we can find $D_{n}$ in $V$ such that $D_{n} \subset C_{n}$ and $\beta_{2}\left(D_{n}\right)<\infty$ and for some $i$,

$$
m<\sum_{n=0}^{i} n \beta_{2}\left(D_{n}\right)<\infty .
$$

Let $E=A \cap\left(X \times \bigcup_{n=0}^{i} D_{n}\right)$. Now, we have

$$
\int \beta_{1}\left(E^{y}\right) d \beta_{2}=\int \beta_{1}\left(A^{y}\right) I_{\cup_{n=0}^{i} D_{n}}(y) d \beta_{2}<\infty .
$$

Hence, since Tonelli's Theorem is valid, $\beta^{*}(E)<\infty$. Since $\beta^{*}(E)>m$, semifiniteness of $\beta^{*}$ follows.

Case 2. Suppose $\beta_{2}(S)=\infty$.

Subcase (i). Suppose $\beta_{2}(B)=0$, where $B$ is as in Case 1. By the semifiniteness of $\beta_{2}$, we can find $G \subset S, G \in V$ and $0<\beta_{2}(G)<\infty$ such that $\int_{G} \beta_{1}\left(A^{y}\right) d \beta_{2}>0$. This is possible since for every $y$ in $S$, $\beta_{1}\left(A^{y}\right)>0$. Now if

$$
G_{n}=\left\{y \in G: \beta_{1}\left(A^{y}\right)<n\right\},
$$

then using the monotone convergence Theorem, we can find $n$ such that $0<\int_{G_{n}} \beta_{1}\left(A^{y}\right) d \beta_{2}<\infty$. Let $E=A \cap\left(X \times G_{n}\right)$. Then since Tonelli's Theorem is valid, $\beta^{*}(E)<\infty$. Clearly,

$$
\beta^{*}(E)=\iint I_{E}(x, y) d \beta_{1} d \beta_{2}=\int_{G_{n}} \beta_{1}\left(A^{y}\right) d \beta_{2}>0 .
$$

Subcase (ii). Suppose $\beta_{2}(B)>0$, where $B$ is as before. Then since $\beta_{2}$ is semifinite, we ran find $C \subset B, C \in V$ and

$$
0<\beta_{2}(C)<\infty \text { and } \int_{C} \beta_{1}\left(A^{y}\right) d \beta_{2}=\infty .
$$

Now, if we replace the set $B$ in the proof of Case 1, Subcase (i) ,by the set $C$ above, the proof of this Subcase follows.

Lemma 4. Let $\beta_{1}$ and $\beta_{2}$ be semifinite. Suppose for every $U \times$ $V$ measurable set $A$, the function $\beta_{1}\left(A^{y}\right)$ is a measurable function of 
y. Then the validity of Tonelli's Theorem implies the semifiniteness of $\beta^{*}$.

Proof. Let $S=\left\{y: \beta_{1}\left(A^{y}\right)>0\right\}$. We separate the proof into two distinct cases. (Here $A$ is as in Lemma 3 ).

Case 1. Suppose $\beta_{2}(B)>0$, where

$$
B=\left\{y: \beta_{1}\left(A^{y}\right)=\infty\right\} .
$$

Let $C \subset B, C \in V$ and $0<\beta_{2}(C)<\infty$. Let $A_{0}=A \cap(X \times C)$, and let the measure $\beta_{3}$ be defined by $\beta_{3}(M)=\beta_{2}(M \cap C)$ for every $M$ in $V$. If $\beta_{1} \times \beta_{3}\left(A_{0}\right)<\infty$, then by Fubini's Theorem,

$$
\infty>\beta_{1} \times \beta_{3}\left(A_{0}\right)=\int \beta_{1}\left(A_{0}^{y}\right) d \beta_{3}=\int_{C} \beta_{1}\left(A^{y}\right) d \beta_{3}=\infty
$$

atnd this is a contradiction. Therefore, $\beta_{1} \times \beta_{3}\left(A_{0}\right)=\infty$. Now we observe that $\int \beta_{3}\left(A_{0 x}\right) d \beta_{1}=\infty$, since, otherwise, we have

$$
\iint I_{A}(x, y) I_{C}(y) d \beta_{2} d \beta_{1}<\infty
$$

and therefore, by the validity of Tonelli's Theorem,

$$
\iint I_{A}(x, y) I_{C}(y) d \beta_{1} d \beta_{2}<\infty
$$

which means that $\int_{C} \beta_{1}\left(A^{y}\right) d \beta_{2}<\infty$, which is a contradiction. If $F=$ $\left\{x: \beta_{3}\left(A_{0 x}\right)>0\right\}$, then $\beta_{1}(F)>0$. Since $\beta_{1}$ is semifinite, we can find $D \in U, D \subset F$ and $0<\beta_{1}(D)<\infty$. Then

$$
0<\int_{D} \beta_{3}\left(A_{0 x}\right) d \beta_{1}<\infty,
$$

which means that

$$
0<\iint I_{A}(x, y) I_{C}(y) I_{D}(x) d \beta_{2} d \beta_{1}<\infty .
$$

Hence if $E=A \cap(D \times C)$, then by the validity of Tonelli's Theorem, $0<\beta^{*}(E)<\infty$.

Case 2. Suppose $\beta_{2}(B)=0$, where $B$ is as above. The proof in this case follows exactly as in the corresponding situation in Lemma 3.

3. In this section, we consider a question of Berberian. In [1, p. 129], the product measure $\beta_{1} \times \beta_{2}$, where $\left(X, U, \beta_{1}\right)$ and $\left(Y, V, \beta_{2}\right)$ 
are any two arbitrary measure spaces as defined in $\S 1$, has been defined to be the unique measure $\pi$ on $U \times V$ having the following two properties:

(i) for every finite measurable rectangle $P \times Q$,

$$
\pi(P \times Q)=\beta_{1}(P) \beta_{2}(Q)
$$

and (ii) for every $A \in U \times V$,

$$
\pi(A)=\sup \{\pi(A \cap(P \times Q))\},
$$

where the supremum is taken over all finite measurable rectangles $P \times Q$. (Here 'finiteness' means that each side of the rectangle has finite measure). When $\beta_{1}$ and $\beta_{2}$ are sigma-finite, $\pi$ coincides with the usual product measure $\beta^{*}$. In fact, Proposition 2 below tells us more than this. In [1, p. 142-3], the proofs of Fubini's and Tonelli's Theorems are given in the sigma-finite case. On p. 144, in Problem 4, Berberian asks the following question:

"What part, if any, of the Fubini theory survives for the product of arbitrary (not necessarily sigma-finite) measures? Does it help to assume that the measures are semifinite?"

In Example 2 and Proposition 4, we answer this question, at least, partly.

Let $\beta^{*}$ and $\beta_{*}$ be the outer and inner measure (see [4], p. 254 and p. 274) induced by the measure $\beta$ (defined in $\S 1$ ). First, we state a few results (omitting their proofs which would be obvious to the serious reader) showing some connections between $\pi, \beta^{*}$ and $\beta_{*}$.

Proposition 1. If $B \in U \times V$ and $\beta^{*}(B)<\infty$, then $\beta^{*}(B)=\pi(B)$. If $\beta_{1}$ and $\beta_{2}$ are semifinite, then $\beta_{*}=\pi$ on $U \times V$.

Proposition 2. If $\beta^{*}$ is semifinite, then $\beta^{*}=\pi$.

Proposition 3. There exists a measure $\beta^{\prime}$ on $U \times V$, taking only the values 0 and $\infty$ such that $\beta^{*}=\pi+\beta^{\prime}$.

Now we consider Fubini's Theorem for the product measure $\pi$. The following Example gives a negative answer even when $\beta_{1}$ and $\beta_{2}$ are semifinite.

Example 2. Consider $\beta_{1}, \beta_{2}, U, V$ and $D$ as in Example 1 in $\S 2$. We note that if $\beta^{*}(P \times Q)<\infty$, then $Q$ is a finite set if $\beta_{1}(P)>0$. This means that $\beta^{*}(D \cap(P \times Q))=0$ for every finite rectangle $P \times Q$. Then $I_{D}(x, y)$ is clearly $\pi$-integrable since $\pi(D)=0$. But the iterated 
integrals of $I_{D}(x, y)$ are not equal.

However, as the following Theorem shows, we can have a partial converse of Fubini's Theorem for measure $\pi$.

Proposition 4. Suppose $f(x, y)$ is a nonnegative $U \times V$-measurable function and either $\iint f d \beta_{2} d \beta_{1}$ or $\iint f d \beta_{1} d \beta_{2}$ is well-defined and finite. Then $f$ is $\pi$-integrable.

Proof. Suppose $\iint f d \beta_{1} d \beta_{2}$ is well-defined and equals $k$ which is finite. Assume that $\int f d \pi=\infty$. We will get a contradiction to this assumption to prove the proposition.

By the monotone convergence theorem, there is a nonnegative simple function $g(x, y)=\sum_{i=1}^{n} c_{i} I_{A_{i}}(x, y)$ such that

$$
g \leqq f \text { and } 2 k<\int g d \pi
$$

By the property (ii) of $\pi$, we can find a finite measurable rectangle $P \times Q$ such that for each $i, 1 \leqq i \leqq n, \pi\left(A_{i}\right)$ is so close to $\pi\left(B_{i}\right)$, where $B_{i}=A_{i} \cap(P \times Q)$, that

$$
k<\int h d \pi<\infty,
$$

where $h(x, y)=\sum_{i=1}^{n} c_{i} I_{B_{i}}(x, y)$. Now we define for every $P^{\prime}$ in $U, Q^{\prime}$ in $V$ and $A$ in $U \times V$, the measures

$$
\begin{aligned}
& \pi_{0}(A)=\pi(A \cap(P \times Q)), \\
& \beta_{3}\left(P^{\prime}\right)=\beta_{1}\left(P^{\prime} \cap P\right) \text { and } \\
& \beta_{4}\left(Q^{\prime}\right)=\beta_{2}\left(Q^{\prime} \cap Q\right) .
\end{aligned}
$$

Since $B_{3}$ and $\beta_{4}$ are both finite measures and since $\pi_{0}$ coincides with $\beta_{3} \times \beta_{4}$ (defined as in $\S 1$ ) on all the $U \times V$ measurable sub-rectangles of $P \times Q$, by the uniqueness of the product measure in this case (see p. 257 in [4]), $\pi_{0}$ is the product measure $\beta_{3} \times \beta_{4}$. Since the function $h$ is $\pi$-integrable, it is also $\pi_{0}$-integrable and so by Fubini's Theorem, we have

$$
k<\int h d \pi=\int h d \pi_{0}=\iint h d \beta_{3} d \beta_{4}
$$

which contradicts that $\iint f d \beta_{1} d \beta_{2}=k$. This proves the Proposition.

\section{REFERENCES}

1. S. K. Berberian, Measure and Integration, The Macmillan Company, New York, 1965. 
2. P. R. Halmos, Measure Theory, D. Van Nastrand Company, Inc., New York, 1950. 3. N. Luther, Unique extension and product measures, Canad. J. Math., 19 (1967), 757-63.

4. H. L. Royden, Real Analysis (second edition), The Macmillan Company (CollierMacmillan Ltd.), London, 1968.

Received February 1, 1971 and in revised form March 15, 1972.

UNIVERSITY OF So. FloridA 



\section{PACIFIC JOURNAL OF MATHEMATICS}

\section{EDITORS}

H. SAMELSON

Stanford University

Stanford, California 94305

C. R. HobBY

University of Washington

Seattle, Washington 98105
J. DUGUNDJI

Department of Mathematics

University of Southern California

Los Angeles, California 90007

RICHARD ARENS

University of California

Los Angeles, California 90024

\section{ASSOCIATE EDITORS}

E. F. BECKENBACH

B. H. NeumanN

F WoLF

K. YoshidA

\section{SUPPORTING INSTITUTIONS}

UNIVERSITY OF BRITISH COLUMBIA

UNIVERSITY OF SOUTHERN CALIFORNIA

CALIFORNIA INSTITUTE OF TECHNOLOGY

UNIVERSITY OF CALIFORNIA

MONTANA STATE UNIVERSITY

STANFORD UNIVERSITY

UNIVERSITY OF NEVADA

NEW MEXICO STATE UNIVERSITY

OREGON STATE UNIVERSITY

UNIVERSITY OF OREGON

OSAKA UNIVERSITY

UNIVERSITY OF TOKYO

UNIVERSITY OF UTAH

WASHINGTON STATE UNIVERSITY

UNIVERSITY OF WASHINGTON

$\stackrel{*}{*} \stackrel{*}{*} \stackrel{*}{*}{ }^{*}{ }^{*}$ MMEICAN MATHEMATICAL SOCIETY

NAVAL WEAPONS CENTER

Printed in Japan by International Academic Printing Co., Ltd., Tokyo, Japan 


\section{Pacific Journal of Mathematics}

\section{Vol. 42, No. $1 \quad$ January, 1972}

Tage Bai Andersen, On Banach space valued extensions from split faces ........

David Marion Arnold, A duality for quotient divisible abelian groups of finite

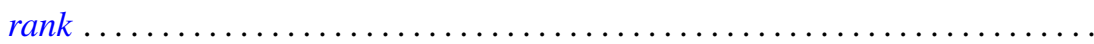

Donald Pollard Ballou, Shock sets for first order nonlinear hyperbolic

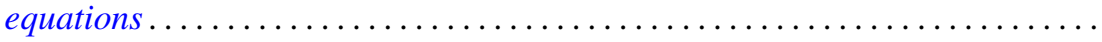

Leon Brown and Lowell J. Hansen, On the range sets of $H^{p}$ functions .........

Alexander Munro Davie and Arne Stray, Interpolation sets for analytic

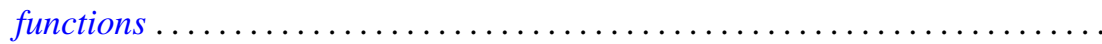

M. G. Deshpande, Structure of right subdirectly irreducible rings. II . . . . . . . . .

Barry J. Gardner, Some closure properties for torsion classes of abelian

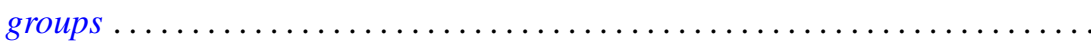

Paul Daniel Hill, Primary groups whose subgroups of smaller cardinality are

direct sums of cyclic groups . . . . . . . . . . . . . . . . . . .

Richard Allan Holzsager, When certain natural maps are equivalences .........

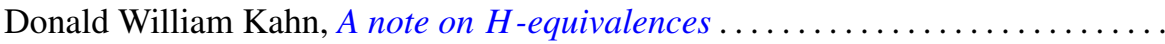

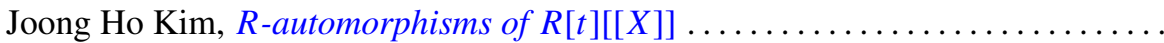

Shin'ichi Kinoshita, On elementary ideals of polyhedra in the 3-sphere.........

Andrew T. Kitchen, Watts cohomology and separability...

Vadim Komkov, A technique for the detection of oscillation of second order

ordinary differential equations .

Charles Philip Lanski and Susan Montgomery, Lie structure of prime rings of characteristic 2

Andrew Lenard, Some remarks on large Toeplitz determinants . .

Kathleen B. Levitz, A characterization of general Z.P.I.-rings. II .

Donald A. Lutz, On the reduction of rank of linear differential systems

David G. Mead, Determinantal ideals, identities, and the Wronskian ...

Arunava Mukherjea, A remark on Tonelli's theorem on integration in product

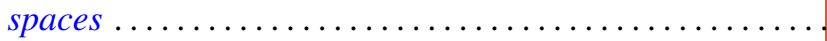

Hyo Chul Myung, A generalization of the prime radical in nonassociative rings.

John Piepenbrink, Rellich densities and an application to unconditionally nonoscillatory elliptic equations.

Michael J. Powers, Lefschetz fixed point theorems for a new class of multi-valued maps .

Aribindi Satyanarayan Rao, On the absolute matrix summability of a Fourier

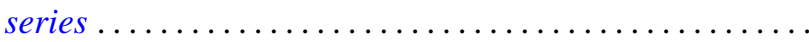

T. S. Ravisankar, On Malcev algebras ......................... 227

William Henry Ruckle, Topologies on sequences spaces . . . . . . . . . . . . . 235

Robert C. Shock, Polynomial rings over finite dimensional rings . . . . . . . . . 251

Richard Tangeman, Strong heredity in radical classes . . . . . . . . . . . . . . 259

B. R. Wenner, Finite-dimensional properties of infinite-dimensional spaces . . . . 267 